\title{
Response measure effects in children as a function of type and schedule of reward'
}

\section{PETER W'ATSON: = Trent University. Peterborough. ()nturio. Canada}

Kindergarten children were administered a series of lever-pulling trials subsequent to either $10 \mathrm{~min}$ or () min social isolation. Lever-pulling responses were reinforced on $0 \% .50 \%$. or 100\% schedules: reinforcements were social ("Good") (ir candy. Isolation had no effect on performance; partial-reviard superiority was demonstrated on a starting-speed measure for social reward. but on a movement-speed measure for cand. reward. The results were interpreted in terms of an hypothesis of frustrative nonreward and S's past history of reinforcement.

It has often been demonstrated (see Ryan \& Watson. 1968) that children receiving partial (e.g., $50 \%$ ) reward for an instrumental response perform faster than when the response is continuously $(100, \mathrm{C})$ rewarded, at least when a tangible reward is employed. Similarly, 50\% reward appears to be optimal, in that both higher and lower percentages produce inferior performance (e.g., Watson, Ryan, \& McEwan. 1967). According to Amsel's (1958) frustrative-nonreward hypothesis. this phenomenon, which may be termed the "partial reward (acquisition) effect." or PRAE. is due to the occurrence of frustration-produced motivation when the organism expects but does not receive reward.

There is a suggestion in the literature (Ryan \& Watson, 1966) that nonattainment of an expected social reward ("Good") may be less frustrating, and thus less motivating. than nonattainment of tangible rewards such as candy. The aim of the present study was to investigate the PRAE in children. using both social and candy rewards. in order to determine whether the frustrative-nonreward hypothesis will prove useful in accounting for social phenomena. Social isolation was included as a variable because of its reputed function as a source of motivation (see Stevenson. 1965). If isolation serves to arouse a social drive, then its effects should be most pronounced in the socially-rewarded Ss: on the other hand, if it arouses a nonspecific drive, the performance of all isolated Ss should be enhanced. On the basis of the assumption that sources of motivation are additive. it was expected that isolation and reward schedule would interact.

\section{METHOD}

The Ss were 71 male and 79 female kindergarten children (mean age = 65.8 months). Immediately prior to the experimental session, half of the Ss received $10 \mathrm{~min}$ of social isolation in a bare room. During the experimental session, Ss were administered a series of 40 lever-pulling trials in which they reached up at the onset of a start light, grasped the lever, and pulled it down through a 15 -in. excursion. Thirty Ss, composing the $0 \%$ reward schedule condition, were not rewarded throughout the 40 trials; the remaining 120 Ss were divided in to four groups which received $100 \%$ social reward, $100 \%$ candy reward, $50 \%$ social reward, and $50 \%$ candy reward. In the socialreward condition, E said "Good" and smiled immediately after completion of each rewarded trial; after rewarded trials in the candy-reward conditions, E rele ased a small candy ("Smarties") which fell into a small box in front of $S$. The intertrial interval was about $8 \mathrm{sec}$, during which $E$ recorded starting time, from the onset of the "start" light to the initial movement of the lever, and movement time, the time taken to pull the lever down through its excursion. The starting and movement times were converted to speeds by means of a reciprocal transformation $(\mathrm{l} / \mathrm{t})$, and combined in to five blocks of eight trials per block. In order to provide a common starting point, the blocked speeds were corrected by dividing by the first-trial starting or movement speed.

\section{RESULTS AND DISCUSSION}

The first point to note concerning the results is that the social isolation had no effects on either starting or movement speeds (all Fs $<2.0$ ). Thus, a 10-min period of social isolation did nut affect the subsequent instrumental performance of the kindergarten Ss used in the present study. This conclusion contrasts with that of Stevenson (1965). who reviews a number of studies which have demunstrated facilitative effects of isolation. Several alternative explanations for the present lack of results might be proposed: perhaps the most likely is that isolation effects are specific to certain types of tasks. certain types of Ss. and/or certain durations of isolation. It may be, for example, that the combination of kindergarten $S$ s and $10 \mathrm{~min}$ of isolation is nonoptimal; Kozma (196\%) has reported similar observations for the combination of Grade $3 \mathrm{Ss}$ and $\mathrm{a}$ 6-min isol.tion period.

The remainder of the results deal with the effects of type and schedule of reward on starting and movement speeds. It should be noted that data of the Origroup were analyzed twice. once in the analyses of the candy-reward condition, and again in the analyses of the social-reward condition. The analyses employed were $T_{y}$.pe $I$ analyses of variance (Lindquist, 1953). with trial blocks as the within-Ss factor and reward schedule as the between-Ss factor.

Figure 1 presents the data involved in the starting speed analyses. For candy reward ( $\mathrm{Fig}$. 1. bottom). the analy sis revealed only a significant main effect for Trial Blocks $(p<.001)$. For social reward (Fig. 1. top). on the other hand. significant effects were obtained for Blocks $(p<.001)$. Reward Schedule $(p<.005)$. and the Blocks by Schedule interaction $(p<.005)$. Analyses of the three social-reward groups taken two at a time suggest that the or group was significantly slower throughuut training than both the $50 r_{r}$ group $(p<.001)$ and the $100 \%$ group $(p<.01)$. In addition. significant Blocks by Schedule interactions in the analyses indicated that the 50 f social-reward group increased as a function of training relative to

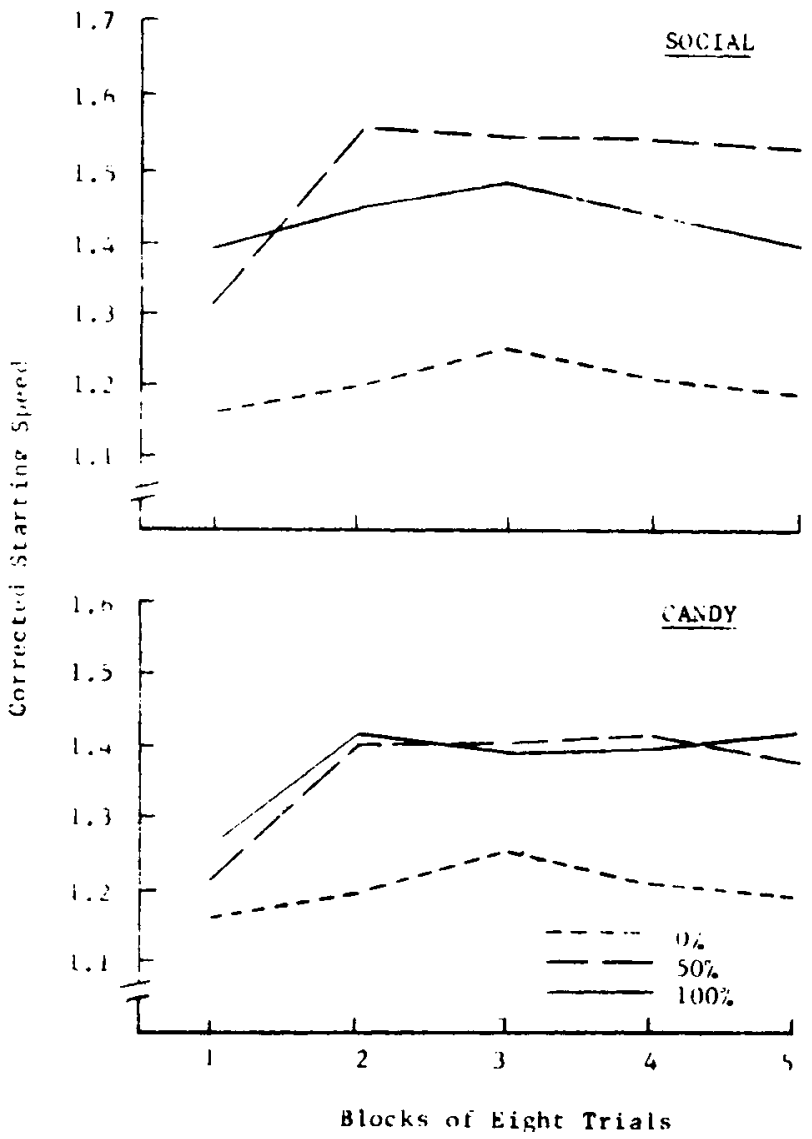

Fig. 1. Mean corrected starting speeds for social-reward (top panel) and candy-reward (bottom panel) groups. as a function of reward schedule and blocks of eight trials. 


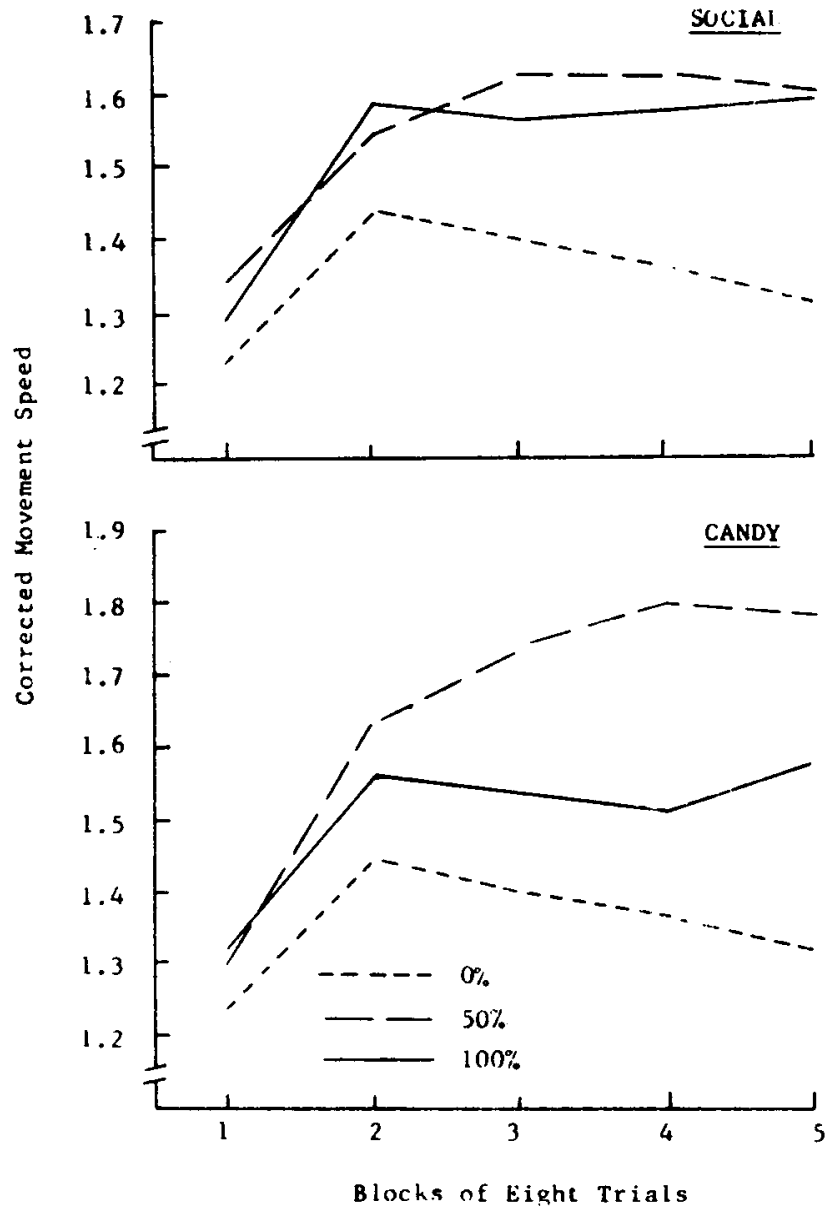

Fig. 2. Mean corrected movement speeds for social-reward (top panel) and candy-reward (bottom panel) groups, as a function of reward schedule and blocks of eight trials.

both the $0 \%(\mathrm{p}<.005)$ and $100 \%$ groups $(\mathrm{p}<.01)$. Thus, it may be concluded that partial social reward leads to increasingly faster starting speed responses, as a function of training, relative to both $100 \%$ social reward and $0 \%$ reward; partial candy reward did not produce a PRAE on starting speeds.

For movement speeds, a different picture obtains (see Fig. 2). For social reward (Fig. 2, top) no significant effects, with the exception of that for Trial Blocks $(\mathrm{p}<.001)$, were obtained. For candy reward (Fig. 2, bot tom) significant effects for Blocks $(\mathrm{p}<.001)$ and for the Block by Schedule interaction $(p<.001)$ were obtained. In order to clarify the interaction, three separate Type 1 analyses compared the $0 \%$ and $50 \%$ groups, the $0 \%$ and $100 \%$ groups, and the $50 \%$ and $100 \%$ groups. Significant Block by Schedule interactions in the two analyses involving the 50\% group suggest that the original interaction was due to movement speeds of the $50 \%$ candy-reward group increasing over training relative to those of the $0 \%$ group $(p<.001)$ and the $100 \%$ candy-reward group $(p<.025)$. These results suggest that the PRAE can be demonstrated on movement speeds when candy reward is employed, and that schedule of social reward does not affect novement speeds.
The pattern apparent in these results is that the PRAE-partial reward superiority, relative to either continuous reward or continuous nonreward -is obtained on a starting speed measure if social reward is employed, and on a movement speed measure if candy reward is employed. It should be noted that these results seem entirely consistent with a number of previous experiments (see Ryan \& Watson, 1968): when tangible rewards such as marbles or candy have been used, 10 of the 11 studies found the PRAE on movement speed, while only four of these studies obtained it on starting speed. In two earlier studies using social reward, the PRAE has only been observed on starting speed. Such results have previously been attributed to experimental error; the present results indicate a fairly high degree of predictability, and at the same time suggest the need for closer consideration of the processes involved in this apparently valid phenomenon.

It may be that the roots of this specificity of the PRAE to particular type-of-reward/response-measure combinations lies in the child's past history of reinforcement by the parents. In spite of a dearth of evidence, it might be assumed that the parents dispense tangible rewards such as candy only on completion of a task, while social rewards are rather more likely to be given during performance of the task. If this is the case, the child may develop an expectancy for certain types of rewards at certain stages of task performance. If it can be assumed that the lever-pulling response as employed in the present study is a prototype of a number of different tasks, the expectancies learned by the child through the reinforcement contin gencies set up by the parents would generalize to the present experimental situation. Frustration in its conditioned form (see Amsel, 1958) would then be evoked only for the response measure appropriate to the type of reward in use.

This interpretation is obviously tentative in the extreme. What is needed at this point is much more information than is presently available on the various psychological processes involved in the starting and movement speed response measures, on the type, frequencies, and sequencing of reinforcement used by parents, and on the complex process whereby frustration can become conditioned to discrete phases of a response sequence.

\section{REFERENCES}

AMSEL, A. The role of frustrative nonreward in noncontinuous reward situations. Psychological Bulletin, 1958, 55, 102-119.

KOZMA, A. The effect of social isolation and visual stimulation on social reinforcer effectiveness. Unpublished doctoral dissertation, University of Western Ontario, 1967.

LINDQUIST, E. F. Design and analy sis of experiments in psychology and education. Boston: Houghton-Mifflin, 1953.

RYAN, T. J., \& WATSON, P. Children's response speeds as a function of sex and verbal reinforcement schedule. Psychonomic Science, 1966, 6, 271-272.

RYAN, T. J., \& WATSON, P. Frustrative nonreward theory applied to children's behavior. Psychological Bulletin, 1968, 69, 111-125.

STEVENSON, H. W. Social reinforcement of children's behavior. In L. P. Lipsitt \& C. C. Spiker (Eds.), Advances in child development and behavior. Vol. 2. New York: Academic Press, 1965.

WATSON, P., RYAN, T. J., \& McEWAN, R. C. Effects of reward schedule and chronological age on motor performance of mental retardates. Psychonomic Science, 1967, 8, 61-62.

\section{NOTES}

1. Based on a Ph.D. dissertation submitted to the Faculty of Graduate Studies of the University of Western Ontario, and supported by a Canada Council Doctoral Fellowship and National Research Council of Canada Grant No. APA-250. The author is grateful to T. J. Ryan and Morton Rieber for valuable advice and to the Board of Education, London, Ontario, for its cooperation in obtaining Ss.

2. Address: Department of Psychology, Trent University, Peterborough, Ontario, Canada. 